\title{
Complete next-to-leading-order calculation for pion production in nucleon-nucleon collisions at threshold
}

\author{
C. Hanhart ${ }^{1}$ and N. Kaiser ${ }^{2}$ \\ ${ }^{1}$ Forschungszentrum Jülich, Institut für Kernphysik (Theorie), D-52425 Jülich, Germany \\ ${ }^{2}$ Institut für Theoretische Physik, Physik Department T39, Technische Universität München, D-85747 Garching, Germany
}

(Received 26 August 2002; published 27 November 2002)

\begin{abstract}
Based on a counting scheme that explicitly takes into account the large momentum $\sqrt{M m_{\pi}}$ characteristic for pion production in nucleon-nucleon collisions we calculate all diagrams for the reaction $N N \rightarrow N N \pi$ at threshold up to next-to-leading-order. At this order there are no free parameters and the size of the next-to-leadingorder contributions is in line with the expectation from power counting. The sum of loop corrections at that order vanishes for the process $p p \rightarrow p p \pi^{0}$ at threshold. The total contribution at next-to-leading-order from loop diagrams that include the delta degree of freedom vanishes at threshold in both reaction channels $p p$ $\rightarrow p p \pi^{0}, p n \pi^{+}$.
\end{abstract}

DOI: 10.1103/PhysRevC.66.054005

PACS number(s): 21.30.Fe, 12.39.Fe, 25.10.+s, 25.40.Ve

The high precision data for the processes $p p \rightarrow p p \pi^{0}$, $p p \rightarrow p n \pi^{+}$and $p p \rightarrow d \pi^{+}$in the threshold region [1] have spurred a flurry of theoretical investigations. The first data on neutral pion production were a big surprise because the experimental cross sections turned out to be a factor of 5 larger than the theoretical predictions based on direct pion production and neutral pion rescattering fixed from on-shell $\pi N$ data $[2,3]$. Subsequently, it was argued that heavy-meson exchanges might be able to remove this discrepancy [4]. On the other hand, it was found [5,6] that the (model-dependent) off-shell behavior of the full $\pi N T$ matrix can also enhance the cross sections near threshold considerably.

Due to their nature as pseudo-Goldstone bosons the dynamics of pions is largely constrained by chiral symmetry. Thus one might hope that effective field theory studies which incorporate these constraints strictly will help to resolve the so far confusing situation. In the literature there are several calculations carried out in the framework of tree-level chiral perturbation theory including the dimension two opeators (single-nucleon) for neutral pion production [7-10] as well as for charged pion production $[11,12]$. A common feature of these calculations is that the contributions from the isoscalar pion rescattering interfere destructively with the direct production amplitude, thus leading to an even more severe discrepancy between experiment and theory. It should be noted that such an interference pattern is in contradiction to the one found in phenomenological approaches [5,6]. Furthermore, within the Weinberg power counting scheme, where all momenta are considered of the order of $m_{\pi}$, one-loop calculations have been performed for neutral pion production $p p$ $\rightarrow p p \pi^{0}[13-15]$. According to some of these works the loop corrections are larger by at least a factor of 2 compared to the tree-level diagrams, which according to the counting scheme applied appear one order down. This feature (if correct) would seriously question the convergence of the chiral expansion for pion production in $N N$ collisions. On the other hand, according to Ref. [16] the chiral expansion seems to show convergence in the case of $p$-wave pion production.

The purpose of the present work is to present a complete next-to-leading-order calculation of the reaction $N N$ $\rightarrow N N \pi$ at threshold. In particular, we evaluate all one-loop diagrams at next-to-leading order employing a counting scheme that takes into account the large momentum $\sqrt{M m_{\pi}}$ characteristic for pion production in $N N$ collisions, as suggested in Refs. $[8,16]$. We consider also the contributions from explicit delta isobars at tree level and at one-loop order. To the order we are working there are no free parameters and we demonstrate that the size of the individual next-toleading-order contributions is in line with the expectations from power counting.

Let us begin with writing down the general form of the threshold $T$ matrix for the pion production reaction $N_{1}(\vec{p})+N_{2}(-\vec{p}) \rightarrow N+N+\pi$ in the center-of-mass frame, which reads [17]

$$
\begin{aligned}
T_{t h}^{\mathrm{cm} .}(N N \rightarrow N N \pi)= & \frac{\mathcal{A}}{2}\left(i \vec{\sigma}_{1}-i \vec{\sigma}_{2}+\vec{\sigma}_{1} \times \vec{\sigma}_{2}\right) \cdot \vec{p} \\
& \times\left(\vec{\tau}_{1}+\vec{\tau}_{2}\right) \cdot \vec{\phi}^{*}+\frac{\mathcal{B}}{2}\left(\vec{\sigma}_{1}+\vec{\sigma}_{2}\right) \cdot \vec{p} \\
& \times\left(i \vec{\tau}_{1}-i \vec{\tau}_{2}+\vec{\tau}_{1} \times \vec{\tau}_{2}\right) \cdot \vec{\phi}^{*}
\end{aligned}
$$

with $\vec{\sigma}_{1,2}$ and $\vec{\tau}_{1,2}$ the spin and isospin operators of the two nucleons. $\vec{\phi}$ denotes the three-component isospin wave function of the final state pion produced in an $s$-wave state, e.g., $\vec{\phi}=(0,0,1)$ for $\pi^{0}$ production and $\vec{\phi}=(1, i, 0) / \sqrt{2}$ for $\pi^{+}$ production. The complex amplitudes $\mathcal{A}$ and $\mathcal{B}$ belong to the transitions ${ }^{3} P_{0} \rightarrow{ }^{1} S_{0}$ and ${ }^{3} P_{1} \rightarrow{ }^{3} S_{1}$ in the two-nucleon system, respectively. In fact the selection rules which follow from the conservation of parity, angular momentum, and isospin allow only for these two transitions for the reaction $N N \rightarrow N N \pi$ at threshold. In the case of neutral pion production $p p \rightarrow p p \pi^{0}$ the threshold amplitude $\mathcal{A}$ is the only relevant one whereas in charged pion production $p p \rightarrow p n \pi^{+}$ both threshold amplitudes $\mathcal{A}$ and $\mathcal{B}$ can contribute. Note that the threshold $T$ matrix written in Eq. (1) incorporates the Pauli exclusion principle since combined left multiplication with the spin-exchange operator $\left(1+\vec{\sigma}_{1} \cdot \vec{\sigma}_{2}\right) / 2$ and the iso- 
spin exchange operator $\left(1+\vec{\tau}_{1} \cdot \vec{\tau}_{2}\right) / 2$ reproduces $T_{t h}^{c m}(N N$ $\rightarrow N N \pi)$ up to an important minus sign. The magnitude of the nucleon center-of-mass momentum $\vec{p}$ necessary to produce a pion at rest is given by

$$
|\vec{p}|=\sqrt{m_{\pi}\left(M+m_{\pi} / 4\right)},
$$

with $M=939 \mathrm{MeV}$ and $m_{\pi}=139.6 \mathrm{MeV}$ denoting the nucleon and pion masses, respectively. Equation (2) exhibits the important feature of the reaction $N N \rightarrow N N \pi$, namely, the large momentum mismatch between the initial and the final nucleon-nucleon state. This leads to a large invariant (squared) momentum transfer $t=-M m_{\pi}$ between in- and outgoing nucleons. The appearance of the large momentum scale $\sqrt{M m_{\pi}}$ in pion production demands for a change in the chiral power counting rules, as pointed out already in Ref. [8]. In addition, it seems compulsory to include the delta isobar as an explicit degree of freedom, since the deltanucleon mass difference $\Delta=293 \mathrm{MeV}$ is comparable to the external momentum $p \simeq \sqrt{M m_{\pi}}=362 \mathrm{MeV}$. The hierarchy of scales

$$
M \gg p \simeq \Delta \gg m_{\pi},
$$

suggested by this feature, is in line with findings within meson exchange models where the delta isobar gives significant contributions even close to the threshold $[18,19]$.

Let us now state our counting rules. The external momentum $p \simeq \sqrt{M m_{\pi}}$ sets the overall scale relevant for the process $N N \rightarrow N N \pi$. This momentum scale $p$ enters the internal lines of tree and loop diagrams. Therefore we count all four-momenta ${ }^{1} l_{\mu}$ inside loops generically as order $p$ and the loop integration measure $\int d^{4} l$ as order $p^{4}$. A pion propagator is counted as order $1 / p^{2}$. The delta propagator of the form $1 /($ energy $-\Delta)$ counts as order $1 / p$, since we made the choice $\Delta \sim p$. For the nucleon propagator of the form 1/energy one has to distinguish whether it occurs outside or inside a loop. The associated residual energy counts as order $m_{\pi}$ outside a loop and as order $p \sim \sqrt{M m_{\pi}}$ inside a loop. Furthermore, external pion energies are counted as order $m_{\pi}$.

According to these counting rules one-loop diagrams contribute at order $p^{2}$ in the expansion of the $T$ matrix and thus generate threshold amplitudes of the form $\mathcal{A}, \mathcal{B} \sim p$ $\simeq \sqrt{M m_{\pi}}$. The new counting rules demand also for a reordering of the terms in the interaction Lagrangian, since "relativistic corrections" proportional to nucleon kinetic energies $p^{2} / M$ are now of the same order as "leading order contributions" proportional to residual nucleon energies. Several examples of this effect will be encountered here.

In Fig. 1, we display tree-level diagrams which according to the above mentioned counting rules contribute at leading order, next-to-leading order, and next-to-next-to-leading order. Diagrams for which the role of both nucleons is interchanged and diagrams with crossed outgoing nucleon lines

\footnotetext{
${ }^{1}$ Baryon energies are residual energies with the nucleon mass $M$ subtracted.
}



a)

b)

c)

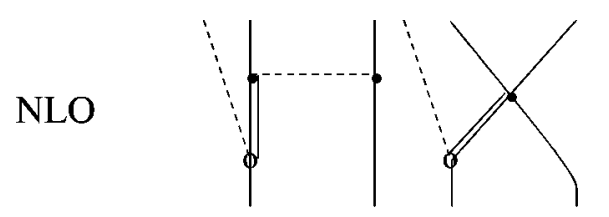

d)

e)

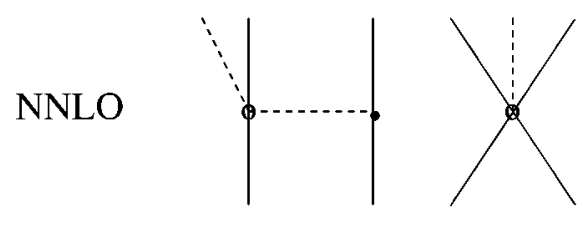

f)

g)

FIG. 1. Tree-level contributions to threshold pion production at leading order (a), (b), and (c), next-to-leading order (d) and (e), and next-to-next-to-leading order (f) and (g). A single solid, double solid, and dashed line denotes a nucleon, delta isobar, and pion, respectively. Leading (subleading) order vertices are symbolized by solid circles (open circles).

are not shown. Subsets of four diagrams obtained by these operations map properly onto the crossing antisymmetric threshold $T$ matrix Eq. (1). Figure 1(a) involving the (isovector) Weinberg-Tomozawa $\pi \pi N N$-contact vertex gives a leading-order contribution of the form

$$
\mathcal{A}^{(W T)}=0, \quad \mathcal{B}^{(W T)}=-\frac{g_{A}}{2 M f_{\pi}^{3}},
$$

with $g_{A} \simeq 1.3$ the nucleon axial vector coupling and $f_{\pi}$ $=92.4 \mathrm{MeV}$ the pion decay constant. It is important to note that the Weinberg-Tomozawa vertex generates here a proportionality factor $m_{\pi}$ at "leading order" in the chiral $\pi N$ Lagrangian via the pion and nucleon (residual) energies as well as through a "relativistic correction" of the form $p^{2} / M$. This factor of $m_{\pi}$ gets finally canceled by the pion propagator $\left[m_{\pi}\left(M+m_{\pi}\right)\right]^{-1}$. Obviously, the isovector WeinbergTomozawa vertex cannot contribute to the neutral pion production threshold amplitude $\mathcal{A}$. From the one-pion exchange in Fig. 1(b) one finds

$$
\mathcal{A}^{(1 \pi)}=\frac{g_{A}^{3}}{8 M f_{\pi}^{3}}, \quad \mathcal{B}^{(1 \pi)}=\frac{3 g_{A}^{3}}{8 M f_{\pi}^{3}} .
$$

This result stems from the recoil correction to the $\pi N N$ vertex proportional to $\left(m_{\pi} / M\right) \vec{\sigma}_{1} \cdot \vec{p}$ with the $m_{\pi}$ factor now getting canceled by the intermediate nucleon propagator. Furthermore, the product of the two vertices on the left nucleon line $\left(\vec{\sigma}_{1} \cdot \vec{p}\right)^{2}=M m_{\pi}$ is canceled by the pion propagator. The ratio $\mathcal{B}^{(1 \pi)} / \mathcal{A}^{(1 \pi)}=3$ has its origin in the isospin 
factor of Fig. 1(b). From the analogous Fig. 1(d) with one virtual delta-isobar excitation one finds

$$
\mathcal{A}^{(\Delta)}=\frac{g_{A}^{3} m_{\pi}}{4 M f_{\pi}^{3} \Delta}, \quad \mathcal{B}^{(\Delta)}=0,
$$

where we have used the empirically well satisfied relation $h_{A}=3 g_{A} / \sqrt{2}$ for the $\pi N \Delta$-coupling constant. The spin and isospin transition operators entering the $\pi N \Delta$ vertex $\left(h_{A} / 2 f_{\pi}\right) \vec{S} \cdot \vec{p} T_{a}$ satisfy the usual relations $S_{i} S_{j}^{\dagger}=\left(2 \delta_{i j}\right.$ $\left.-i \epsilon_{i j k} \sigma_{k}\right) / 3$ and $T_{a} T_{b}^{\dagger}=\left(2 \delta_{a b}-i \epsilon_{a b c} \tau_{c}\right) / 3$. The latter isospin relation is the reason behind the vanishing of $\mathcal{B}^{(\Delta)}$. According to our counting of the mass splitting $\Delta$ the term $\mathcal{A}^{(\Delta)}$ in Eq. (6) is a next-to-leading-order contribution, since $\Delta \sim p$ [cf. relation (3)]. Figure 1(f) involves the second-order chiral $\pi \pi N N$-contact vertex proportional to the low-energy constants $c_{1,2,3,4}$ [20]. We find the following contributions to the threshold amplitudes at next-to-next-to-leading order:

$$
\begin{gathered}
\mathcal{A}^{\left(c_{i}\right)}=\frac{g_{A} m_{\pi}}{2 M f_{\pi}^{3}}\left(c_{3}+2 c_{2}-4 c_{1}\right), \\
\mathcal{B}^{\left(c_{i}\right)}=\frac{g_{A} m_{\pi}}{2 M f_{\pi}^{3}}\left(c_{4}+c_{3}+2 c_{2}-4 c_{1}\right) .
\end{gathered}
$$

In a previous calculation in Ref. [7] [see Eq. (32) therein] the $c_{2}$ term has been found with a relative factor $1 / 2$ smaller. The reason for this discrepancy is again that "relativistic corrections" from the $c_{2}$ vertex are of the same order as its "static" contribution, since $p^{2} / M=m_{\pi}$. We also note that our results, Eqs. (4)-(7) agree up to the respective order with those of the fully relativistic calculation in Ref. [17] where no approximations to the threshold kinematics have been made. We do not specify the contributions from diagrams (c), (e), and $(\mathrm{g})$ in Fig. 1 which are proportional to the (a priori unknown) strengths of four-nucleon contact vertices, etc. It is important to note that already at leading order long-range effects from pion-exchange and short-range contributions appear simultaneously.

Let us now turn to the nonvanishing one-loop diagrams at threshold. Not every loop diagram appearing formally at next-to-leading order truly contributes at that order. In the case of diagrams (b) and (c) in Fig. 2 the (spin-independent) one-loop $\pi N$-scattering subdiagrams are proportional to $m_{\pi}^{3}$, and this pushes their contributions to the threshold $T$-matrix Eq. (1) beyond next-to-leading order. A closer inspection of diagram (a) in Fig. 2 reveals that they contribute in the form $m_{\pi} \ln m_{\pi}$ to the threshold amplitudes $\mathcal{A}$ and $\mathcal{B}$, i.e., beyond next-to-leading order. The specific vertex structures of diagrams (d) and (e) in Fig. 2 make also their next-to-leadingorder contributions vanishing. Therefore we have to focus only on the diagrams shown in Figs. 3 (and 4).

We evaluate only the genuine next-to-leading-order pieces of the loop integrals emerging from the diagrams in Figs. 3 (and 4). For instance, in the integrands we can systematically drop terms of order $m_{\pi}$ compared to $l_{0}$ (and $\Delta$ ). Straightfor-

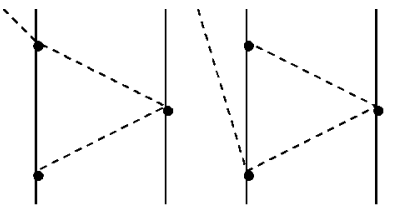

a)

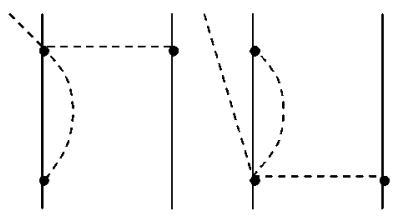

c)

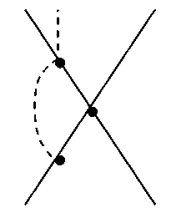

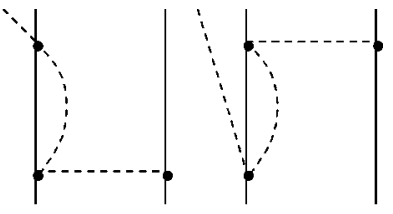

b)



d)

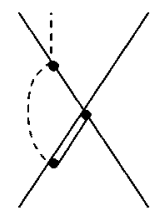

e) f)

FIG. 2. One-loop diagrams that start to contribute at next-tonext-to-leading order. For further notations see Fig. 1.

ward but tedious evaluation leads to the following next-toleading-order contributions of the one-loop diagrams in Fig. 3 with nucleons only:

$$
\begin{aligned}
& \mathcal{A}^{(N-\text { loop })}=\frac{g_{A}^{3} \sqrt{M m_{\pi}}}{256 f_{\pi}^{5}}(-2-1+3), \\
& \mathcal{B}^{(N-\text { loop })}=\frac{g_{A}^{3} \sqrt{M m_{\pi}}}{256 f_{\pi}^{5}}(-2+0+3) .
\end{aligned}
$$

Here, the numerical entries correspond to the diagrams (a), (b), and (c) of Fig. 3, in that order. Interestingly, the total next-to-leading-order loop contribution vanishes identically for neutral pion production $\mathcal{A}^{(N-\text { loop })}=0$. The diagrams (a) and (c) in Fig. 3 have been calculated fully relativistically (i.e., without any approximation to the threshold kinematics) for $p p \rightarrow p p \pi^{0}$ in Ref. [17]. It is an important check for our calculation that the nonanalytical piece proportional to $\sqrt{M m_{\pi}}$ agrees with the one derived by expanding Eq. (16) in Ref. [17]. In addition, after correcting a sign error in Ref.

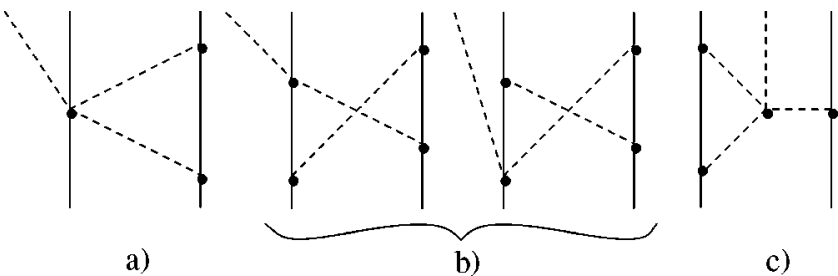

FIG. 3. Next-to-leading-order one-loop diagrams for pion production at threshold with nucleons only. For further notations see Fig. 1. 


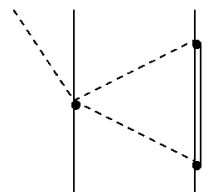

a)

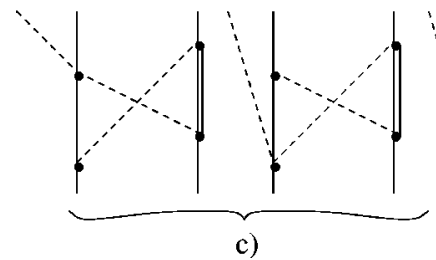

FIG. 4. Next-to-leading-order one-loop diagrams for pion production at threshold with intermediate delta isobars. For further notations see Fig. 1.

[14] and extracting at threshold the truly next-to-leadingorder pieces from that work our results agree with theirs [21].

Numerically, the loop correction in Eq. (8) gives $\mathcal{B}^{(N-\text { loop })}=g_{A}^{3} \sqrt{M m_{\pi}} / 256 f_{\pi}^{5} \simeq 0.70 \mathrm{fm}^{4}$. This is about $50 \%$ of the leading order one-pion exchange contributions $\left|\mathcal{B}^{(W T)}\right| \simeq 1.33 \mathrm{fm}^{4}$ or $\mathcal{B}^{(1 \pi)} \simeq 1.69 \mathrm{fm}^{4}$. Indeed from chiral power counting one expects a similar suppression factor $p / M=\sqrt{m_{\pi} / M} \simeq 0.4$.

According to our counting of the mass difference $\Delta$ $\sim \sqrt{M m_{\pi}}$ loop diagrams with explicit delta isobars are of the same order as those with nucleons only, namely, of order $p^{2}$. The relevant one-loop diagrams which generate truly nextto-leading-order contributions are shown in Fig. 4. Straightforward but tedious evaluation leads to the following result:

$$
\begin{aligned}
& \mathcal{A}^{(\Delta-\text { loop })}=\frac{g_{A}^{3} K(\Delta)}{32 f_{\pi}^{5}}(8-12+1+3), \\
& \mathcal{B}^{(\Delta-\text { loop })}=\frac{g_{A}^{3} K(\Delta)}{32 f_{\pi}^{5}}(8-12+3+1)
\end{aligned}
$$

with the numerical entries corresponding to the subclasses (a), (b), (c), and (d) of Fig. 4, in that order. The relevant combination of loop functions reads

$$
\begin{aligned}
K(\Delta)= & 2 J_{0}(-\Delta)-2 \Delta I_{0}\left(-M m_{\pi}\right)+\left(2 \Delta^{2}-M m_{\pi}\right) \\
& \times \gamma_{0}\left(-\Delta,-M m_{\pi}\right)
\end{aligned}
$$

with the following loop integrals [20] truncated at lowest order according to our counting scheme:

$$
\begin{aligned}
& J_{0}(-\Delta)=4 \Delta L(\lambda)+\frac{\Delta}{4 \pi^{2}}\left(\ln \frac{2 \Delta}{\lambda}-\frac{1}{2}\right) \sim \mathcal{O}(p) \\
& I_{0}\left(-M m_{\pi}\right)=-2 L(\lambda)-\frac{1}{16 \pi^{2}}\left(1+\ln \frac{M m_{\pi}}{\lambda^{2}}\right) \sim \mathcal{O}\left(p^{0}\right),
\end{aligned}
$$

$$
\begin{aligned}
\gamma_{0}\left(-\Delta,-M m_{\pi}\right) & =\frac{1}{4 \pi^{2} \sqrt{M m_{\pi}}} \int_{0}^{\infty} \frac{d x}{1+x^{2}} \arctan \frac{x \sqrt{M m_{\pi}}}{2 \Delta} \\
& \sim \mathcal{O}\left(p^{-1}\right)
\end{aligned}
$$

The (scale-dependent) quantity

$$
L(\lambda)=\frac{\lambda^{d-4}}{16 \pi^{2}}\left[\frac{1}{d-4}+\frac{1}{2}\left(\gamma_{E}-1-\ln 4 \pi\right)\right]
$$

denotes for the standard divergent piece in dimensional regularization. The reason for grouping together the three specific diagrams into subclass (b) is that this way the (in the chiral limit) singular term $\Delta^{2} J_{0}(-\Delta) / M m_{\pi}$ does not appear explicitly. Evidently, the formal limit $\Delta \rightarrow 0$ corresponds to loop diagrams with nucleons only, and therefore $K(0)=$ $-\sqrt{M m_{\pi}} / 16$ enters Eq. (8). Note, however, that for planar box diagrams this limit becomes inconsistent with the counting scheme employed.

One concludes that the contributions stemming from loop diagrams with delta excitation vanish identically for both threshold amplitudes $\mathcal{A}$ and $\mathcal{B}$, respectively, for both reaction channels $p p \rightarrow p p \pi^{0}, p n \pi^{+}$. The complete cancellations in Eq. (9) are actually important consistency checks for our power counting scheme $\Delta \sim p$. The combination of loop functions $K(\Delta) \sim p$ in Eq. (10) is divergent, but at next-toleading order there is no local counter term to absorb divergences.

In summary, we have performed a complete next-toleading-order calculation of the reaction $N N \rightarrow N N \pi$ at threshold. We have employed the counting scheme developed in Refs. [8,16], that explicitly accounts for the large momentum $p \simeq \sqrt{M m_{\pi}}$ characteristic for this process. We find that the total next-to-leading-order loop corrections either vanish or are in accordance with the expectation from power counting. At this stage we conclude that the chiral expansion seems to converge also in the $s$ wave. Note, however, that at next-to-next-to-leading order a large number of loops enters that has not yet been evaluated completely.

In order to compare our results directly to pion production data the emerging chiral operators have to be folded with (realistic) $N N$-wave functions. This convolution has been carried out in Ref. [15] in such a way that the symmetries are preserved. However, in that work the traditional Weinberg counting has been used. Consequently the results presented in Ref. [15] do not allow any firm conclusion about the convergence of the chiral series, since contributions of different orders are mixed and the next-to-next-to-leading order is incomplete. Based on our counting scheme a complete next-tonext-to-leading-order calculation is within reach and should be performed. Another direction should be the calculation of loop corrections to the higher partial wave amplitudes. 
[1] For a recent review see H. Machner and J. Haidenbauer, J. Phys. G 25, R231 (1999).

[2] G.A. Miller and P. Sauer, Phys. Rev. C 44, 1725 (1991).

[3] J.A. Niskanen, Phys. Lett. B 289, 227 (1992).

[4] T.-S.H. Lee and D.O. Riska, Phys. Rev. Lett. 70, 2237 (1993).

[5] E. Hernandez and E. Oset, Phys. Lett. B 350, 158 (1995).

[6] C. Hanhart, J. Haidenbauer, A. Reuber, C. Schütz, and J. Speth, Phys. Lett. B 358, 21 (1995).

[7] B.Y. Park, F. Myhrer, J.R. Morones, T. Meissner, and K. Kubodera, Phys. Rev. C 53, 1519 (1996).

[8] T.D. Cohen, J.L. Friar, G.A. Miller, and U. van Kolck, Phys. Rev. C 53, 2661 (1996).

[9] U. van Kolck, G.A. Miller, and D.O. Riska, Phys. Lett. B 388, 679 (1996).

[10] T. Sato, T.-S.H. Lee, F. Myhrer, and K. Kubodera, Phys. Rev. C 56, 1246 (1997).

[11] C. Hanhart et al., Phys. Lett. B 424, 8 (1998).
[12] C. da Rocha, G.A. Miller, and U. van Kolck, Phys. Rev. C 61, 034613 (2000).

[13] E. Gedalin, A. Moalem, and L. Razdolskaya, Phys. Rev. C 60, 031001(R) (1999).

[14] V. Dmitrasinovic, K. Kubodera, F. Myhrer, and T. Sato, Phys. Lett. B 465, 43 (1999).

[15] S. Ando, T.S. Park, and D.P. Min, Phys. Lett. B 509, 253 (2001).

[16] C. Hanhart, U. van Kolck, and G.A. Miller, Phys. Rev. Lett. 85, 2905 (2000).

[17] V. Bernard, N. Kaiser, and Ulf-G. Meißner, Eur. Phys. J. A 4, 259 (1999).

[18] J.A. Niskanen, Phys. Rev. C 53, 526 (1996).

[19] C. Hanhart, J. Haidenbauer, O. Krehl, and J. Speth, Phys. Lett. B 444, 25 (1998).

[20] V. Bernard, N. Kaiser, and Ulf-G. Meißner, Int. J. Mod. Phys. E 4, 193 (1995).

[21] F. Myhrer (private communication). 